\title{
Is the Location of the Tumor Another Prognostic Factor for Patients With Colon Cancer?
}

\author{
Myung-Kyu Jung, Ui Sup Shin, Young-Jun Ki, Yong-Bae Kim, Sun-Mi Moon, Se-Jin Sung \\ Department of Surgery, Korea Cancer Center Hospital, Korea Institute of Radiological \& Medical Sciences, Seoul, Korea
}

Purpose: In this study, we investigated both the characteristics of right colon cancer (RTCC) in comparison with those of left colon cancer (LTCC) and the impact of the location of the colon cancer on the prognosis.

Methods: We retrospectively analyzed the cases of 974 patients with nonmetastatic colon cancer who had undergone surgery with a curative intent from January 2001 to December 2011. RTCC was defined as a tumor located proximal to the splenic flexure. The characteristics of RTCC cancer were investigated by using descriptive analyses, and their impacts on the prognosis were assessed by using a Cox multivariate regression.

Results: Compared to LTCC, RTCC showed a female-dominant feature, and an undifferentiated pathology was more frequently observed. The number of lymph nodes retrieved from patients with RTCC was significantly higher than that retrieved from patients with LTCC. During 75 months of follow-up, peritoneal recurrence was more common in patients with RTCC than it was in patients with LTCC, and among the patients with stage III colon cancer, the disease-free and the overall survival rates were significantly worse in patients with RTCC. After adjustments with the other prognostic factors associated with colon cancer had been made, a tumor located at the right colon was found to be independently associated with poor prognosis.

Conclusion: RTCC showed unique clinicopathologic features and was associated with a poorer prognosis.

Keywords: Colon adenocarcinoma; Survival; Location; Prognosis

\section{INTRODUCTION}

In the year 2013, 27,618 patients out of a total 225,343 cancer patients (12.3\%) were diagnosed with colorectal cancer; it is the second most common malignancy in men and the third in women according to a report by the Korean National Cancer Information Center [1]. If the colon is split into right and left portions based on the splenic flexure, each part is different in its embryological origin and shows different physiologic features. Accordingly, different carcinogenetic pathways between the 2 colonic segments

Received: July 27, 2017 - Accepted: September 24, 2017

Correspondence to: Ui Sup Shin, M.D.

Department of Surgery, Korea Cancer Center Hospital, Korea Institute of Radiological \& Medical Sciences, 75 Nowon-ro, Nowon-gu, Seoul 01812, Korea

Tel: +82-2-9701216, Fax: +82-2-9702419, E-mail: uisupshin@kirams.re.kr

(c) 2017 The Korean Society of Coloproctology

This is an open-access article distributed under the terms of the Creative Commons Attribution NonCommercial License (http://creativecommons.org/licenses/by-nc/4.0) which permits unrestricted noncommercial use, distribution, and reproduction in any medium, provided the original work is properly cited. have been suggested, and many differences in the clinical, pathological, and genetic features according to the sidedness of the colon cancer have been reported. The following differences have been noted: proximal colon cancers are more likely to be diploid and to have mucinous histology, a high level of microsatellite instability (MSI), CpG island methylation, and BRAF mutation whereas left-sided colon cancers are often infiltrating lesions having chromosomal instability and are more often aneuploidy [2-4]. Although which of these biologic differences according to tumor location translate into meaningful differences in prognosis is still unclear, in recent studies, the location of the primary tumor has been emphasized for its prognostic and predictive role, with worse outcome being associated with right-sided colon cancer with distant metastasis [5-9]. However, in regard to patients who have undergone a curative resection of nonmetastatic colon cancer, the prognostic role of the primary tumor's location is still a subject of debate [10-15]. For this reason, the aim of this study was to analyze the clinicopathological differences between rightand left-sided colon cancers and to evaluate the impacts of the sidedness of the primary tumor on recurrence and survival after a 
curative resection of nonmetastatic colon cancer.

\section{METHODS}

We retrospectively analyzed 974 consecutive patients who had undergone a curative resection between January 2001 and December 2011 at a single institution, Korea Cancer Center Hospital, after having been diagnosed with colon cancer. All patients were identified from a prospectively collected institutional colorectal cancer database. We included patients with a pathologically proven adenocarcinoma at the colon whose distal margins were proximally located to anal verge (AV), $15 \mathrm{~cm}$ at flexible endoscopy. Patients with mucosal cancer, distant metastasis, hereditary colon cancer, or previous history of malignancy were excluded. Patients with synchronous colon cancer located on both the right and the left side of the colon were also excluded. Right colon cancer (RTCC) was defined as cancer with the tumor located proximal to the splenic flexure whereas left colon cancer (LTCC) was defined as cancer with the tumor located between the splenic flexure and AV $15 \mathrm{~cm}$.

All patients underwent curative surgery after a preoperative staging workup including colonoscopy, computed tomography (CT), and positron emission tomography. Pathologic examinations were performed by pathologists who were dedicated to the treatment of patients with gastrointestinal cancer and who used the 7th American Joint of Cancer Staging system. In addition to TNM information, data regarding lymphatic, vascular or perineural invasion, differentiation of the tumor, and histologic type were also collected.

After recovery from surgery, adjuvant treatment was recommended to all medically fit patients whose cancers were identified as stage II or stage III on pathologic examinations. The FOLFOX (5-fluorouracil, oxaliplatin, leucovorin) regimen was recommended to all stage III patients. However, stage II patients were stratified according to the following features: high risk of recurrence, including obstruction or perforation at the primary tumor; pathologic T4 stage; poorly differentiated or mucinous/signet ring cell patterns; lymphovascular or perineural invasion. The FOLFOX regimen was recommended to high-risk stage II patients, depending on their medical fitness for undergoing adjuvant chemotherapy. According to institutional policy, the FL regimen was recommended to stage II patients without any high-risk features. However, for patients exhibiting a high level of MSI or loss of the protein MLH1 or MSH2 on immunohistochemistry tests, although such tests were not performed routinely, adjuvant chemotherapy was not recommended. Posttreatment surveillance was performed using physical examinations and evaluations of the carcinoembryonic antigen level, with or without cross-sectional imaging, every 3-6 months for the first 2 years and then annually thereafter.

Statistical analyses were performed using the R software package ver. 3.3.1 (R Foundation for Statistical Computing, Vienna, Aus- tria). Descriptive statistics was used to analyze the clinical and the pathologic parameters according to the location of the colon cancer. Categorical variables were compared using the chi-square or Fisher exact test. An independent sample t-test or Wilcoxon rank sum test was used according to their normality.

For the survival analysis, the Kaplan-Meier method was used. For the comparison of the survival results between the location groups, the log rank test was used. For the determination of the independency of the location of the colon cancer as a prognostic factor, a multivariate Cox regression with step-wise backward elimination was performed with other possible prognostic parameters. For the multivariate regression, variables whose P-values less than 0.5 on the univariate analyses were chosen. In the calculation of the disease-free survival (DFS), an event was defined as any kind of recurrence or any cause of death. In all cases, a P-value less than 0.05 was considered as statistically significant.

\section{RESULTS}

Among the identified 974 patients with colon cancer, 329 (33.8\%) had RTCC and 645 (66.2\%) had LTCC. The median age of the patients was 63 years (interquartile range [IQR], $54-70$ years), and the male-to-female ratio was 1.3:1. One hundred thirty (13.3\%) were found to have stage I cancer, 436 (43.7\%) stage II cancer, and 418 (42.9\%) stage III cancer. When the ages of patients with LTCC and RTCC were compared, no statistically significant difference in median age was found. However, the frequency of female patients was significantly higher in patients with RTCC. The stage distributions and the kinds of adjuvant treatment did not differ between the 2 location groups. Neither did the frequencies of lymphatic, vascular, and perineural invasion. However, the frequency of pathologic T4 stage cancer and the median number of total retrieved lymph node were significantly higher in patients with RTCC. Also, poor prognostic histologic features, including mucinous or signet ring cell features, were more frequently identified among the patients with RTCC (Table 1).

During the median 74-month follow-up (IQR, 50- to 110month follow-up), 154 recurrences (15.8\%) and 169 deaths (17.3\%) were observed. Among the 154 patients who experienced a recurrence, the liver was the most common relapse site, and the lungs were the second. Peritoneal recurrence was identified in 22 patients (2.6\%). Among the 22 patients with a peritoneal recurrence, 15 (15 of 329, 4.6\%) had RTCC, which significantly outnumbered the 7 with LTCC $(\mathrm{P}=0.001)$. No other significant differences at the site of the recurrence according to the location of the primary tumor were noted (Table 2).

According to the Kaplan-Meier analyses, the 5-year DFS and the overall survival (OS) of all patients were $80.0 \%$ and $86.7 \%$, respectively. When the 5-year DFSs were compared according to pathologic stage, no difference between patients with stage I and II RTCC and those with stage I and stage II LTCC were found. However, in the stage III subgroup, RTCC patients showed a sig- 
Table 1. Descriptive analyses according to the location of the colon cancer

\begin{tabular}{|c|c|c|c|}
\hline Variable & RTCC $(n=329)$ & LTCC $(n=645)$ & $P$-value \\
\hline Sex & & & 0.007 \\
\hline Male & $167(50.8)$ & $387(60.0)$ & \\
\hline Female & $162(49.2)$ & $258(40.0)$ & \\
\hline Age (yr) & $64.0(52.0-71.0)$ & $63.0(55.0-70.0)$ & 0.994 \\
\hline Stage & & & 0.554 \\
\hline I & $43(13.1)$ & 87 (13.5) & \\
\hline$\|$ & $137(41.6)$ & $289(44.8)$ & \\
\hline III & $149(45.3)$ & $269(41.7)$ & \\
\hline No. total LN & $22.0(16.0-31.0)$ & $15.0(10.0-21.0)$ & $<0.001$ \\
\hline Pathologic LN & $0(0-2.0)$ & $0(0-2.0)$ & 0.217 \\
\hline pT4 & 50 (15.2) & $60(9.3)$ & 0.008 \\
\hline PD/MUC/SRC & $48(15.0)$ & $26(4.1)$ & $<0.001$ \\
\hline Lymphatic invasion & $128(38.9)$ & 255 (39.5) & 0.904 \\
\hline Perineural invasion & 60 (18.2) & $136(21.1)$ & 0.335 \\
\hline Vascular invasion & 41 (12.5) & $62(9.6)$ & 0.209 \\
\hline Obstruction & $29(8.8)$ & $67(10.4)$ & 0.506 \\
\hline Perforation & $4(1.2)$ & $9(1.4)$ & 1.000 \\
\hline Adjuvant treatment, yes & $250(76.0)$ & $521(80.8)$ & 0.098 \\
\hline \multicolumn{4}{|l|}{ Stage II } \\
\hline $\mathrm{FL}$ & 120/137 (87.6) & 259/289 (89.6) & 0.150 \\
\hline FOLFOX & $11 / 137(8.0)$ & 26/289 (9.0) & \\
\hline None & 6/137 (4.4) & 4/289 (1.4) & \\
\hline \multicolumn{4}{|l|}{ Stage III } \\
\hline $\mathrm{FL}$ & $60 / 149(40.3)$ & $122 / 269(45.4)$ & 0.600 \\
\hline FOLFOX & 69/149 (46.3) & $114 / 269(42.4)$ & \\
\hline None & 20/149 (13.4) & $33 / 269$ (12.3) & \\
\hline
\end{tabular}

Values are presented as number (\%) or median (interquartile range).

RTCC, right colon cancer; LTCC, left colon cancer; LN, lymph node; PD, poor differentiation; MUC, mucinous; SRC, signet ring cell; FL, 5-fluorouracil, leucovorin; FOLFOX, 5-fluorouracil, oxaliplatin, leucovorin.

nificantly worse DFS than the LTCC patients did ( $58 \%$ vs. $72.7 \%$, $\mathrm{P}=0.01$ ) (Fig. 1). The 5 -year OS of stage III patients also showed a significant difference between the location groups (66.6\% RTCC vs. $82.3 \%$ LTCC, $P=0.0026$ ), even though no significant differences were found for the patients in the stage I and the stage II subgroups (Fig. 2)

To investigate the prognostic impact of the location of the colon cancer, we performed a Cox regression analysis with other clinical and pathological variables. The univariate analysis for DFS revealed that the location of the colon cancer on the right side was associated with increased risk of recurrence or death (hazard ratio [HR], 1.21; 95\% confidence interval [CI], 0.92-1.58), as were other clinicopathologic factors without statistical significance.
Table 2. Patterns of recurrence according to the location of the colon cancer

\begin{tabular}{lccc}
\hline Variable & RTCC $(\mathrm{n}=329)$ & LTCC $(\mathrm{n}=645)$ & P-value \\
\hline Recurrence & $55(16.7)$ & $99(15.3)$ & 0.645 \\
Site of recurrence & & & \\
$\quad$ Liver & $18(5.5)$ & $35(5.4)$ & 1.000 \\
Lung & $12(3.6)$ & $33(5.1)$ & 0.384 \\
Peritoneum & $15(4.6)$ & $7(1.1)$ & 0.001 \\
Distant lymph node & $15(4.6)$ & $27(4.2)$ & 0.917 \\
Bone & $2(0.6)$ & $1(0.2)$ & 0.552 \\
Ovary & $3(0.9)$ & $2(0.3)$ & 0.442 \\
Local recurrence & $3(0.9)$ & $6(0.9)$ & 1.000 \\
Others & $4(1.2)$ & $4(0.6)$ & 0.549 \\
\hline
\end{tabular}

Values are presented as number (\%).

However, after a multivariate analysis, having adjusted for other clinicopathologic factors, a cancer located on the right side of the colon was significantly associated with poor prognosis (adjusted HR [aHR], 1.49; 95\% CI, 1.11-2.0; P = 0.009) (Table 3). In the Cox regression analysis for OS, cancer located on the right side of the colon constantly showed significant associations with OS on both the uni- and the multivariate analyses (aHR, 1.64; 95\% CI, $1.15-2.36 ; \mathrm{P}=0.007$ ) (Table 4).

\section{DISCUSSION}

In this single-institution retrospective study, we found that patients with RTCC showed distinct clinical and pathological features, including an increased frequency of pathologic T4 stage cancer and a mucinous or poorly differentiated histology. Also, patients with RTCC experienced more frequent peritoneal recurrence than patients with LTCC did. Although, only patients with RTCC in the stage III subgroup showed significant worse survival, in the multivariate analyses, a location of the primary tumor on the right side of the colon was an independent, poor prognostic factor for recurrence-free and OS. Although many controversies exist concerning the impact of the location of colon cancer on prognosis, a steady series of reports have stated that the location of the colon cancer does affect the prognosis. Recently, the response to antiepidermal growth factor receptor (anti-EGFR) treatment has been reported to be worse in patients with metastatic RTCC. At the 2016 American Society of Clinical Oncology meeting, Venook et al. retrospectively evaluated data from the phase III, CALGB/SWOG 80405 clinical trial, a clinical trial designed to compare bevacizumab and cetuximab concurrent with chemotherapy as initial therapy for patients with metastatic colorectal cancer $[6,16]$. They analyzed 293 patients with RTCC and 732 patients with LTCC. Those with a tumor on the left side had longer median OS (33.3 months) than those with a tumor on 
Stage I

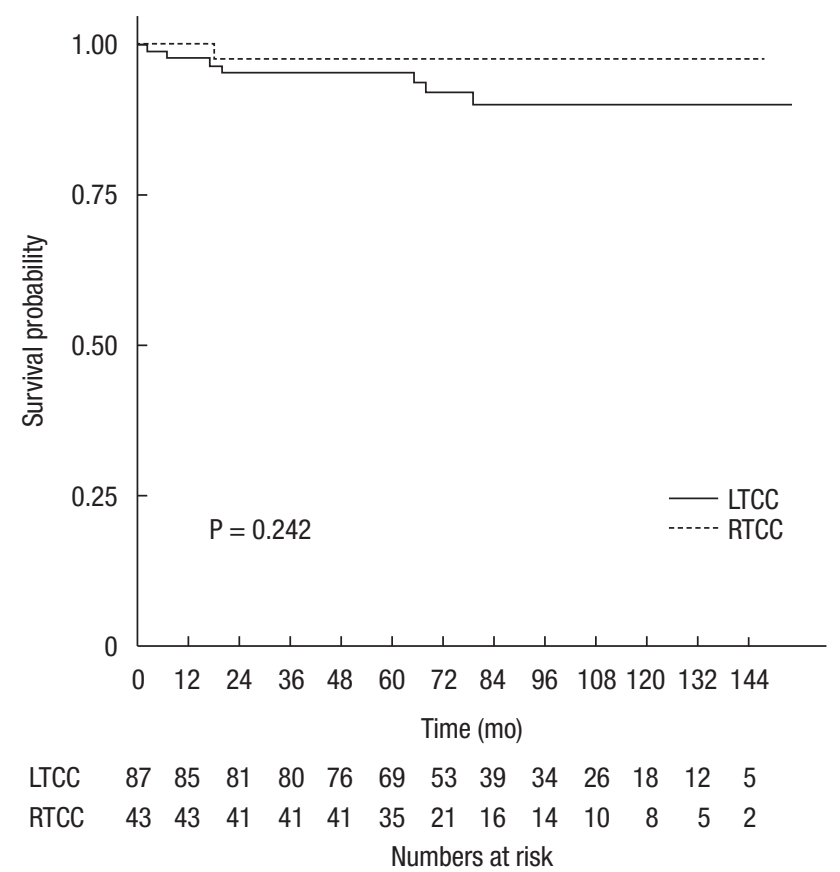

Stage III

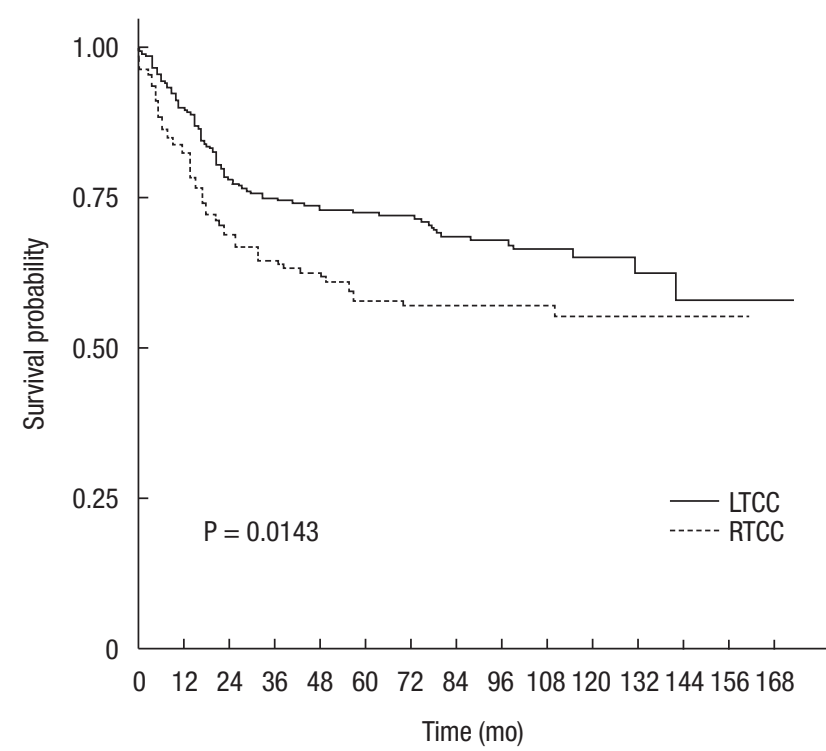

LTCC $\quad 26923920419418616313211195 \quad 70 \quad 41 \quad 20 \quad 10 \quad 4 \quad 2$

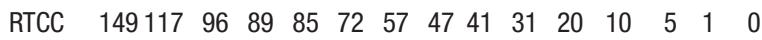
Numbers at risk

the right side (19.4 months) [5]. Regardless of the kind of antiEGFR agent, similar trends of inferior survival for patients with RTCC were observed in other retrospective studies that had analyzed the results of previous randomized controlled studies $[7,17$, 18].

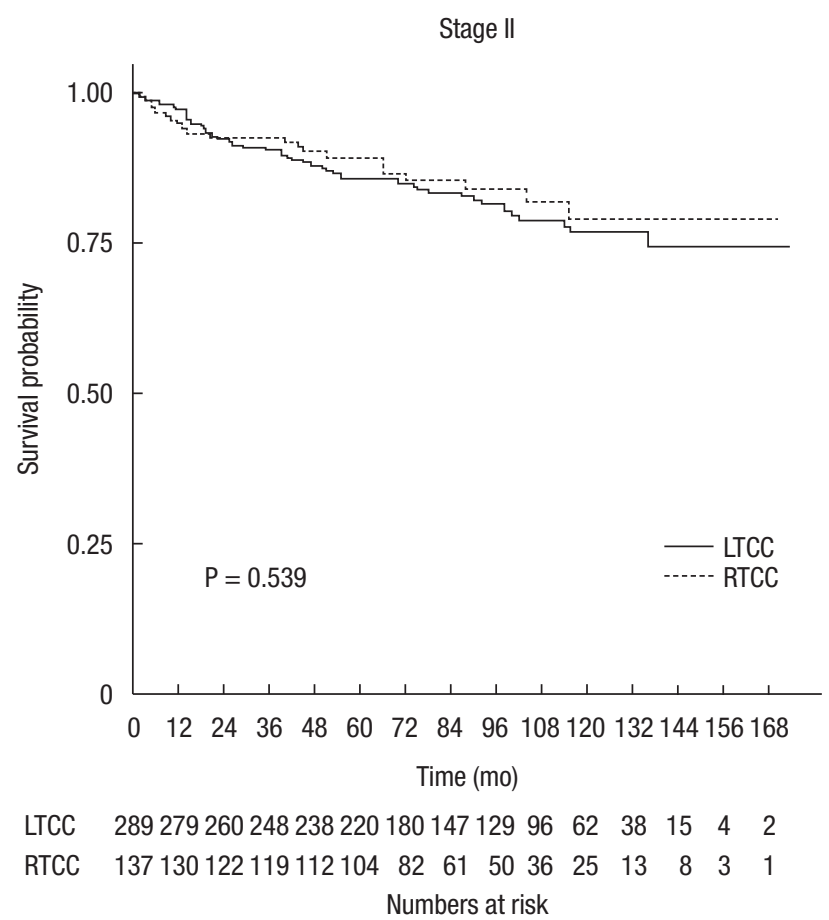

Fig. 1. Disease-free survival according to the location of the colon cancer at pathologic stages I, II, and III. LTCC, left colon cancer; RTCC, right colon cancer.

However, in studies involving patients with nonmetastatic colon cancer, a poorer outcome for patients with RTCC was not constantly observed among the stages, as was the case in studies involving patients with metastatic cancers $[10,12,15]$. Recently, the results of 3 large-scale population-based studies involving 17,000 


\section{Coloproctology
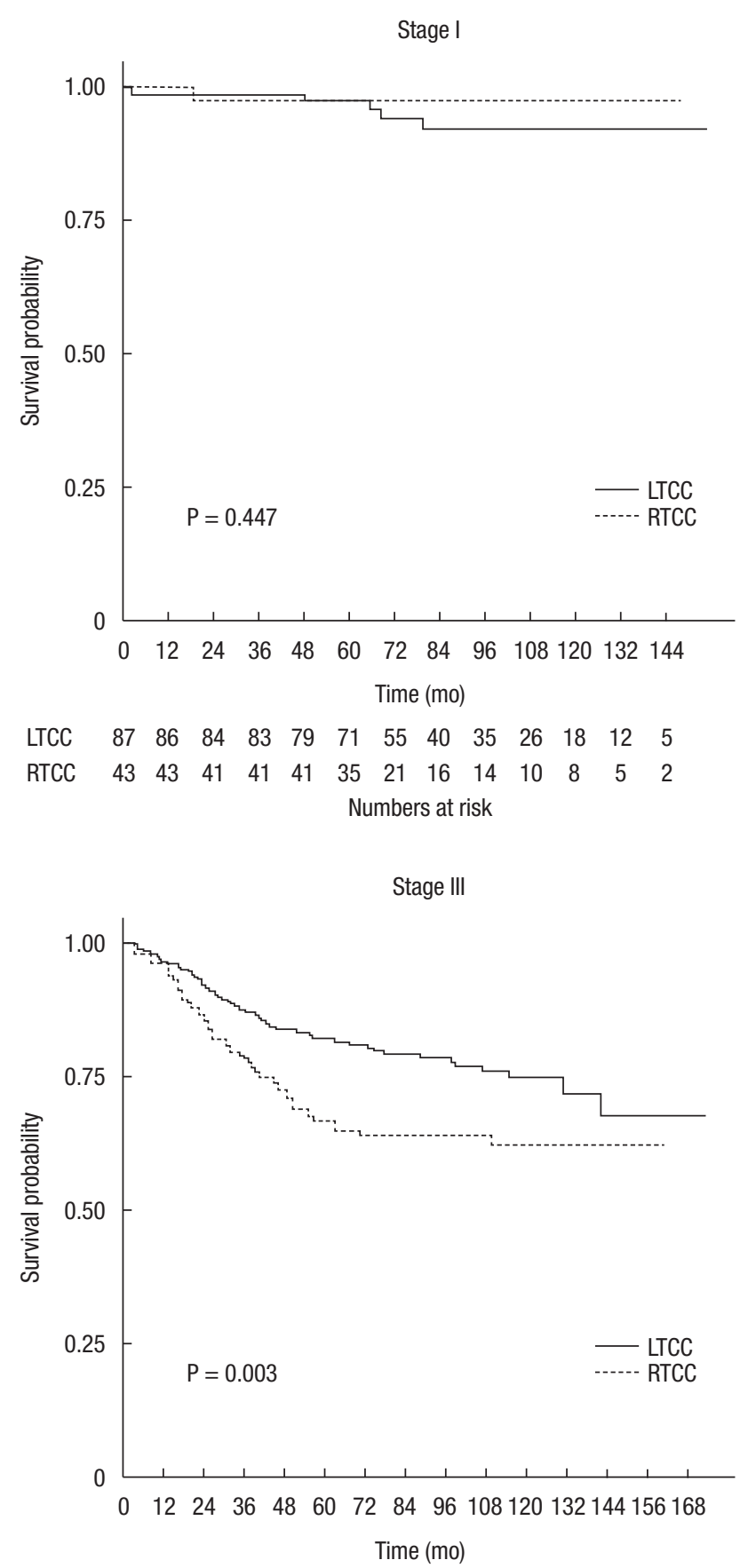

LTCC $\quad 269257241226213186149131108 \quad 77 \quad 46 \quad 23 \quad 12 \quad 4 \quad 2$

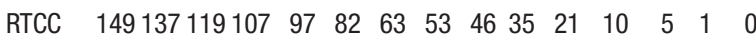
Numbers at risk

to 90,000 people with nonmetastatic colon cancer were reported. The first study showed a higher 5-year overall mortality (67\% vs. $71 \%, \mathrm{P}<0.01)$ and a lower DFS ( $73 \%$ vs. $74 \%, \mathrm{P}<0.01)$ for patients with RTCC. In subanalyses of survival according to stage, only patients with stage I and III cancer showed a significant dif-

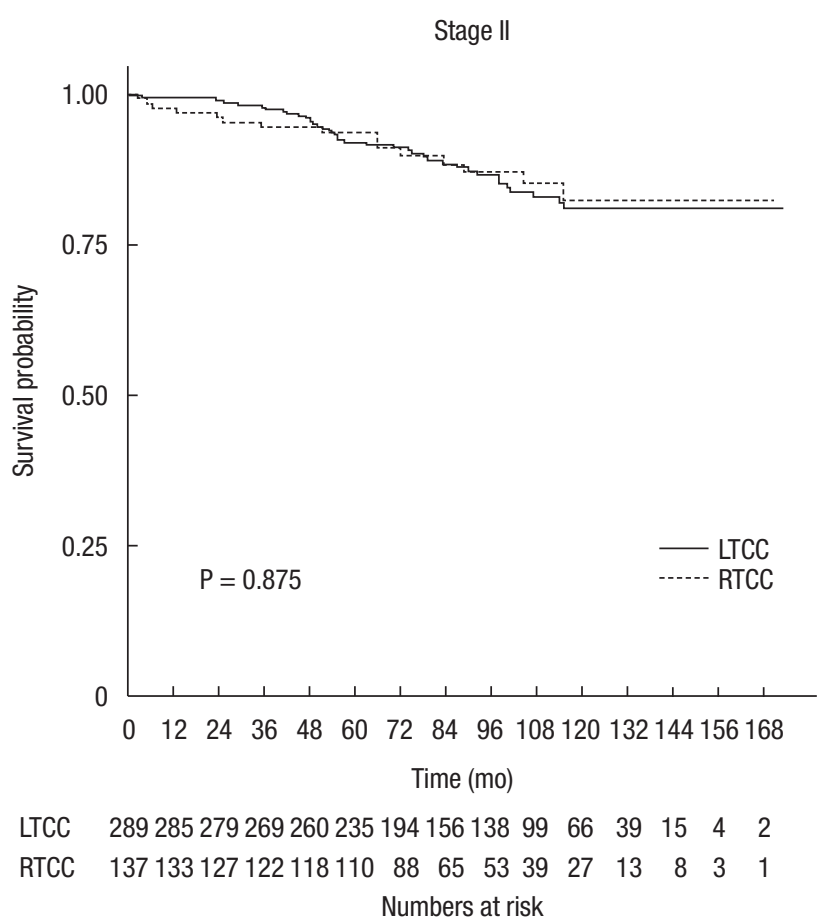

Fig. 2. Overall survival according to the location of the colon cancer at pathologic stages I, II, and III. LTCC, left colon cancer; RTCC, right colon cancer.

ference in OS, and no difference according to stage was observed in the DFS [10]. The second study revealed a higher mortality risk for patients with stage III cancer located on the right side of the colon after adjustment for another covariate (HR, 1.12; 95\% CI, 1.06-1.18; $\mathrm{P}<0.001$ ) [15]. The last study examined the largest 
Table 3. Cox regression analyses for prognostic factors of disease-free survival

\begin{tabular}{|c|c|c|c|c|}
\hline \multirow{2}{*}{ Factor } & \multicolumn{2}{|c|}{ Univariate } & \multicolumn{2}{|c|}{ Multivariate } \\
\hline & $\mathrm{HR}(95 \% \mathrm{Cl})$ & P-value & $\mathrm{HR}(95 \% \mathrm{Cl})$ & P-value \\
\hline Male sex & $1.15(0.88-1.50)$ & 0.305 & $1.25(0.94-1.65)$ & 0.122 \\
\hline Age & $1.02(1.01-1.03)$ & 0.001 & $1.02(1.00-1.03)$ & 0.015 \\
\hline Stage I & 1 & & 1 & - \\
\hline Stage II & $2.90(1.40-6.02)$ & 0.004 & 4.32 (1.99-9.37) & $<0.001$ \\
\hline Stage III & $6.89(3.38-14.05)$ & $<0.001$ & $6.20(2.80-13.71)$ & $<0.001$ \\
\hline Total examined LN & $0.99(0.98-1.00)$ & 0.107 & $0.97(0.96-0.99)$ & 0.001 \\
\hline Pathologic LN & $1.12(1.09-1.16)$ & $<0.001$ & $1.09(1.05-1.14)$ & $<0.001$ \\
\hline pT4 & $1.81(1.27-2.57)$ & 0.001 & - & - \\
\hline $\mathrm{PD} / \mathrm{MUC} / \mathrm{SRC}$ & $2.27(1.55-3.34)$ & $<0.001$ & - & - \\
\hline Lymphatic invasion & $2.40(1.84-3.12)$ & $<0.001$ & $1.42(1.06-1.92)$ & 0.021 \\
\hline Perineural invasion & $2.18(1.65-2.88)$ & $<0.001$ & $1.49(1.10-2.01)$ & 0.010 \\
\hline Vascular invasion & $2.80(2.03-3.86)$ & $<0.001$ & $1.61(1.14-2.28)$ & 0.006 \\
\hline Obstruction & $2.05(1.45-2.90)$ & $<0.001$ & $1.50(1.04-2.16)$ & 0.031 \\
\hline Perforation & $3.40(1.68-6.88)$ & 0.001 & - & - \\
\hline Adjuvant treatment & $0.75(0.55-1.02)$ & 0.065 & $0.49(0.34-0.69)$ & $<0.001$ \\
\hline Right colon cancer & $1.21(0.92-1.58)$ & 0.169 & $1.49(1.11-2.00)$ & 0.009 \\
\hline
\end{tabular}

$\mathrm{HR}$, hazard ratio; Cl, confidence interval; LN, lymph node; PD, poor differentiation; MUC, mucinous; SRC, signet ring cell.

Table 4. Cox regression analyses for prognostic factors of overall survival

\begin{tabular}{|c|c|c|c|c|}
\hline \multirow{2}{*}{ Factor } & \multicolumn{2}{|c|}{ Univariate } & \multicolumn{2}{|c|}{ Multivariate } \\
\hline & $\mathrm{HR}(95 \% \mathrm{Cl})$ & P-value & HR $(95 \% \mathrm{Cl})$ & P-value \\
\hline Male sex & $1.15(0.84-1.57)$ & 0.374 & $1.34(0.96-1.86)$ & 0.085 \\
\hline Age & $1.04(1.02-1.06)$ & $<0.001$ & $1.03(1.02-1.05)$ & $<0.001$ \\
\hline Stage I & 1 & - & 1 & - \\
\hline Stage II & $2.70(1.16-6.29)$ & 0.021 & $4.16(1.70-10.17)$ & 0.002 \\
\hline Stage III & $6.38(2.80-14.53)$ & $<0.001$ & 5.16 (2.06-12.92) & $<0.001$ \\
\hline Total examined LN & $0.99(0.97-1.00)$ & 0.093 & $0.97(0.95-0.99)$ & $<0.001$ \\
\hline Pathologic LN & $1.12(1.08-1.15)$ & $<0.001$ & $1.11(1.05-1.17)$ & $<0.001$ \\
\hline pT4 & $1.65(1.08-2.52)$ & 0.021 & - & - \\
\hline PD/MUC/SRC & $3.06(2.04-4.59)$ & $<0.001$ & $1.73(1.09-2.72)$ & 0.019 \\
\hline Lymphatic invasion & 2.59 (1.89-3.54) & $<0.001$ & $1.45(1.01-2.06)$ & 0.042 \\
\hline Perineural invasion & $2.23(1.61-3.08)$ & $<0.001$ & $1.63(1.14-2.31)$ & 0.007 \\
\hline Vascular invasion & $2.89(2.01-4.17)$ & $<0.001$ & $1.59(1.08-2.35)$ & 0.019 \\
\hline Obstruction & $2.31(1.57-3.40)$ & $<0.001$ & $1.54(1.03-2.32)$ & 0.036 \\
\hline Perforation & 2.66 (1.09-6.49) & 0.031 & $0.45(0.30-0.67)$ & $<0.001$ \\
\hline Adjuvant treatment & $0.59(0.42-0.84)$ & 0.003 & $0.45(0.30-0.67)$ & $<0.001$ \\
\hline Right colon cancer & $1.45(1.06-1.97)$ & 0.020 & $1.64(1.15-2.36)$ & 0.007 \\
\hline
\end{tabular}

HR, hazard ratio; Cl, confidence interval; LN, lymph node; PD, poor differentiation; MUC, mucinous; SRC, signet ring cell.

population, more than 90,000 colon cancer patients. The propensity score matching technique was applied to adjust for other con- founders. Before the matching, patients with RTCC showed worse survival, as expected. However, after matching, the survival inferi- 
ority for patients with RTCC disappeared; rather, patients with RTCC showed superior survival overall (HR, 0.92; 95\% CI, 0.890.94; $\mathrm{P}<0.001$ ) and superior cancer-specific survival (HR, 0.9; 95\% CI, 0.87-0.93; P < 0.001) [12]. Those studies did not report any constant result of an inferior survival for patients with RTCC of any stage. However, common findings of those studies were that the survival rates of patients with RTCC were worse than those of patients with LTCC, especially for patients in stage III, and that with stage escalation, the decrease in the survival rate for patients with RTCC was more prominent than it was for patients with LTCC. Our study showed similar trends. In the stages I and II subgroup, the DFS and the OS did not differ according to the sidedness of the colon cancer, but in the stage III subgroup, patients with RTCC showed significantly worse survival.

Although the reasons patients with RTCC show worse survival are still unclear, regarding the poor survival of patients with RTCC, especially those in an advanced stage, gene alterations that accumulate, along with the progression of colon cancer, might differ according to the cancer's location $[19,20]$. The colonic segment proximal to the splenic flexure (right-sided colon) and the distal segment (left-sided colon) are of different embryological origins [3]. Also, significant variations exist at the molecular level between right- and left-sided colon cancer, which may serve as the causes of all apparent differences in the clinical, pathological, and biological features [21-24]. Among the various genotypes related to right-sided colon cancer, the MSI is a well-known representative feature of RTCC, which has been shown to have a favorable impact on the prognosis for patients who undergo a curative resection of stage II colorectal cancer [25]. Although MSI was not present, the superior outcomes for patients with RTCC in stage II in some studies may reflect these features of RTCC $[12,15,26]$. The BRAF is another component in the RAS-RAF-MAPK signal pathway, with a reported incidence of $2.5 \%-20 \%$ in patients with colorectal cancer [27, 28]. BRAF mutation is known to have a close relationship to right-sided colon cancer and its poor prognosis [29-31]. Although BRAF mutations tend to coexist with MSI through their relationship with the high-level CpG island methylator phenotype and MLH1 promoter methylation, colon cancers with $B R A F$ mutations showed dismal outcomes, regardless of MSI status [30,32,33]. Colon cancers with BRAF mutations have distinct clinicopathological features, including being frequently observed in proximal colon cancer and more commonly having a mucinous, signet ring cell, or serrated pathology. Also, colon cancers with BRAF mutations are related to more frequent peritoneal carcinomatosis [32,33]. Although we found no relationship between the BRAF or the MSI status and our findings, those molecular features might be possible clues to an explanation for our results of frequent peritoneal carcinomatosis and poor prognosis for patients with RTCC.

The latest NCCN guidelines [34], based on the previously mentioned studies regarding the poor response to the use of the antiEGFR agent for the treatment of patients with RTCC, recom- mend its use as a first-line treatment only for patients with LTCC $[5,6,8,9]$. In regard to patients with nonmetastatic colon cancer, even though much evidence exists for the clinical impact of the sidedness of colon cancer on the prognosis, additional research is still needed if different therapeutic approaches are to be applied according to the location of the tumor.

As a limitation of our study, due to its' retrospective nature, we were unable to obtain information about the genetic status of the tumor regarding MSI or BRAF, which might be a possible clue to an explanation of our findings. During the study period, our institution did not routinely perform the MSI test or immunohistochemistry staining for mismatch repair proteins such as MLH1/ MSH2. Therefore, over treatments of stage II patients with a rightsided tumor, who might have had MSI-H features, may have existed, and those treatments may have had an effect on the worse outcome observed for patients with RTCC. However, comparing the frequency of MSI-H with the Western countries, that of MSI$\mathrm{H}$ in advanced stages was relatively low based on domestic data. Therefore, we presumed that the confounding effect of MSI-H might be little [35]. Another limitation of this study is the possible existence of additional confounders, such as the surgeons' experiences, the patients' comorbidities, and treatment after recurrence; those factors represent unmeasured confounders and may be the cause of a possible bias.

In summary, despite the limitations of this retrospective study, we found that the prognosis for patients with colon cancer was significantly affected by the location of the tumor, especially for patients with cancer in an advanced stage. We hope that our results would be a little step to lead the future researches to find the specific molecular markers which associated to our findings.

\section{CONFLICT OF INTEREST}

No potential conflict of interest relevant to this article was reported.

\section{REFERENCES}

1. Jung KW, Won YJ, Kong HJ, Oh CM, Lee DH, Lee JS. Prediction of cancer incidence and mortality in Korea, 2014. Cancer Res Treat 2014;46:124-30.

2. Bufill JA. Colorectal cancer: evidence for distinct genetic categories based on proximal or distal tumor location. Ann Intern Med 1990;113:779-88.

3. Gervaz P, Bucher P, Morel P. Two colons-two cancers: paradigm shift and clinical implications. J Surg Oncol 2004;88:261-6.

4. Shen H, Yang J, Huang Q, Jiang MJ, Tan YN, Fu JF, et al. Different treatment strategies and molecular features between right-sided and left-sided colon cancers. World J Gastroenterol 2015;21:64708.

5. Venook AP, Niedzwiecki D, Innocenti F, Fruth B, Greene C, O'Neil $\mathrm{BH}$, et al. Impact of primary tumor location on overall survival 
(OS) and progression-free survival (PFS) in patients (pts) with metastatic colorectal cancer (mCRC): analysis of CALGB/SWOG 80405 (Alliance) [abstract]. In: 2016 ASCO Annual Meeting; 2016 Jun 3-7; Chicago (IL), USA. Alexandria (VA): American Society of Clinical Oncology; 2016. Abstract No. 3504.

6. Schrag D, Weng S, Brooks G, Meyerhardt JA, Venook AP. The relationship between primary tumor sidedness and prognosis in colorectal cancer [abstract]. In: 2016 ASCO Annual Meeting; 2016 Jun 3-7; Chicago (IL), USA. Alexandria (VA): American Society of Clinical Oncology; 2016. Abstract No. 3505.

7. Arnold D, Lueza B, Douillard JY, Peeters M, Lenz HJ, Venook A, et al. Prognostic and predictive value of primary tumour side in patients with RAS wild-type metastatic colorectal cancer treated with chemotherapy and EGFR directed antibodies in six randomized trials. Ann Oncol 2017;28:1713-29.

8. Brulé SY, Jonker DJ, Karapetis CS, O’Callaghan CJ, Moore MJ, Wong R, et al. Location of colon cancer (right-sided versus leftsided) as a prognostic factor and a predictor of benefit from cetuximab in NCIC CO.17. Eur J Cancer 2015;51:1405-14.

9. Chen KH, Shao YY, Chen HM, Lin YL, Lin ZZ, Lai MS, et al. Primary tumor site is a useful predictor of cetuximab efficacy in the third-line or salvage treatment of KRAS wild-type (exon 2 nonmutant) metastatic colorectal cancer: a nationwide cohort study. BMC Cancer 2016;16:327.

10. Benedix F, Kube R, Meyer F, Schmidt U, Gastinger I, Lippert H, et al. Comparison of 17,641 patients with right- and left-sided colon cancer: differences in epidemiology, perioperative course, histology, and survival. Dis Colon Rectum 2010;53:57-64.

11. Ishihara S, Nishikawa T, Tanaka T, Tanaka J, Kiyomatsu T, Kawai $\mathrm{K}$, et al. Prognostic impact of tumor location in stage IV colon cancer: a propensity score analysis in a multicenter study. Int J Surg 2014;12:925-30.

12. Warschkow R, Sulz MC, Marti L, Tarantino I, Schmied BM, Cerny T, et al. Better survival in right-sided versus left-sided stage I - III colon cancer patients. BMC Cancer 2016;16:554.

13. Powell AG, Wallace R, McKee RF, Anderson JH, Going JJ, Edwards J, et al. The relationship between tumour site, clinicopathological characteristics and cancer-specific survival in patients undergoing surgery for colorectal cancer. Colorectal Dis 2012;14: 1493-9.

14. Yahagi M, Okabayashi K, Hasegawa H, Tsuruta M, Kitagawa Y. The worse prognosis of right-sided compared with left-sided colon cancers: a systematic review and meta-analysis. J Gastrointest Surg 2016;20:648-55.

15. Weiss JM, Pfau PR, O’Connor ES, King J, LoConte N, Kennedy G, et al. Mortality by stage for right- versus left-sided colon cancer: analysis of surveillance, epidemiology, and end results--Medicare data. J Clin Oncol 2011;29:4401-9.

16. CALGB/SWOG C80405: A phase III trial of FOLFIRI or FOLFOX with bevacizumab or cetuximab or both for untreated metastatic adenocarcinoma of the colon or rectum. Clin Adv Hematol Oncol 2006;4:452-3.
17. Tejpar S, Stintzing S, Ciardiello F, Tabernero J, Van Cutsem E, Beier F, et al. Prognostic and predictive relevance of primary tumor location in patients with RAS wild-type metastatic colorectal cancer: retrospective analyses of the CRYSTAL and FIRE-3 trials. JAMA Oncol 2016 Oct 10 [Epub]. https://doi.org/10.1001/jamaoncol.2016.3797.

18. Moretto R, Cremolini C, Rossini D, Pietrantonio F, Battaglin F, Mennitto A, et al. Location of primary tumor and benefit from anti-epidermal growth factor receptor monoclonal antibodies in patients with RAS and BRAF wild-type metastatic colorectal cancer. Oncologist 2016;21:988-94.

19. Yokota J. Tumor progression and metastasis. Carcinogenesis 2000;21:497-503.

20. Worthley DL, Whitehall VL, Spring KJ, Leggett BA. Colorectal carcinogenesis: road maps to cancer. World J Gastroenterol 2007; 13:3784-91.

21. Nawa T, Kato J, Kawamoto H, Okada H, Yamamoto H, Kohno H, et al. Differences between right- and left-sided colon cancer in patient characteristics, cancer morphology and histology. J Gastroenterol Hepatol 2008;23:418-23.

22. Lindblom A. Different mechanisms in the tumorigenesis of proximal and distal colon cancers. Curr Opin Oncol 2001;13:63-9.

23. Missiaglia E, Jacobs B, D’Ario G, Di Narzo AF, Soneson C, Budins$\mathrm{ka} \mathrm{E}$, et al. Distal and proximal colon cancers differ in terms of molecular, pathological, and clinical features. Ann Oncol 2014;25: 1995-2001.

24. Azzoni C, Bottarelli L, Campanini N, Di Cola G, Bader G, Mazzeo A, et al. Distinct molecular patterns based on proximal and distal sporadic colorectal cancer: arguments for different mechanisms in the tumorigenesis. Int J Colorectal Dis 2007;22:115-26.

25. Merok MA, Ahlquist T, Røyrvik EC, Tufteland KF, Hektoen M, Sjo $\mathrm{OH}$, et al. Microsatellite instability has a positive prognostic impact on stage II colorectal cancer after complete resection: results from a large, consecutive Norwegian series. Ann Oncol 2013;24:1274-82.

26. Brungs D, Aghmesheh M, de Souza P, Ng W, Chua W, Carolan M, et al. Sidedness is prognostic in locoregional colon cancer: an analysis of 9509 Australian patients. BMC Cancer 2017;17:251.

27. Chen D, Huang JF, Liu K, Zhang LQ, Yang Z, Chuai ZR, et al. BRAFV600E mutation and its association with clinicopathological features of colorectal cancer: a systematic review and metaanalysis. PLoS One 2014;9:e90607.

28. Yaeger R, Cercek A, Chou JF, Sylvester BE, Kemeny NE, Hechtman JF, et al. BRAF mutation predicts for poor outcomes after metastasectomy in patients with metastatic colorectal cancer. Cancer 2014;120:2316-24.

29. Yamauchi M, Morikawa T, Kuchiba A, Imamura Y, Qian ZR, Nishihara R, et al. Assessment of colorectal cancer molecular features along bowel subsites challenges the conception of distinct dichotomy of proximal versus distal colorectum. Gut 2012;61: 847-54.

30. Pai RK, Jayachandran P, Koong AC, Chang DT, Kwok S, Ma L, et al. BRAF-mutated, microsatellite-stable adenocarcinoma of the 


\section{Coloproctology

proximal colon: an aggressive adenocarcinoma with poor survival, mucinous differentiation, and adverse morphologic features. Am J Surg Pathol 2012;36:744-52.

31. Eklöf V, Wikberg ML, Edin S, Dahlin AM, Jonsson BA, Öberg Å, et al. The prognostic role of KRAS, BRAF, PIK3CA and PTEN in colorectal cancer. Br J Cancer 2013;108:2153-63.

32. Tran B, Kopetz S, Tie J, Gibbs P, Jiang ZQ, Lieu CH, et al. Impact of BRAF mutation and microsatellite instability on the pattern of metastatic spread and prognosis in metastatic colorectal cancer. Cancer 2011;117:4623-32.

33. Jang MH, Kim S, Hwang DY, Kim WY, Lim SD, Kim WS, et al. BRAF-mutated colorectal cancer exhibits distinct clinicopatho- logical features from wild-type BRAF-expressing cancer independent of the microsatellite instability status. J Korean Med Sci 2017;32:38-46.

34. Clinical Practice Guidelines in Oncology (NCCN Guidelines ${ }^{\circledR}$ ), Colon Cancer Version 2. 2017 [Internet], Fort Wathington (PA): National Comprehensive Cancer Network; c2017 [cited 2017 Mar 13]. Available from: https://www.nccn.org/professionals/physician_gls/pdf/colon_blocks.pdf.

35. Oh JR, Kim DW, Lee HS, Lee HE, Lee SM, Jang JH, et al. Microsatellite instability testing in Korean patients with colorectal cancer. Fam Cancer 2012;11:459-66. 